\title{
Choice of Power and Control Hardware for Smart LED Luminary
}

\author{
A. Suzdalenko ${ }^{1}$, I. Galkin ${ }^{2}$ \\ ${ }^{1}$ RTU, Kronvalda boul.1, LV1010 Riga, Latvia,E-mail: Aleksandrs.Suzdalenko@RTU.lv \\ ${ }^{2}$ RTU, Kronvalda boul.1, LV1010 Riga, Latvia,E-mail: gia@avene.eef.rtu.lv
}

\begin{abstract}
This paper analyzes the most significant details of development of a modern luminary. One of the effective ways to improve the energy efficiency of lighting systems and luminaries is to use the so called smart luminaries. This means that the luminaries turn on exactly at the place and time when the light is required, as well as with the required intensity of light. Such approach is especially effective if LED elements are utilized. Development of the smart LED luminaries includes several tasks: elaboration of energy efficient power supply equipped with distance controllable dimming function; choice and implementation of sensors; choice and development of communications for data exchange between luminaries, sensors and overall control system. The paper briefly describes the main problems of development of the smart lighting systems with LEDs as well as their solutions.
\end{abstract}

\section{Introduction}

Generally the concept of Smart Grid means enhancement of common electric grid with monitoring, analysis, control and communication capabilities in order to improve reliability, maximize throughput, increase energy efficiency, provide consumer participation in the energy distribution as well as allow diverse generation and storage options.

The devices of Smart Grid tend to be efficient in energy consumption field as well as "smart" enough to be able to decide whether the device is working usefully or not. A good example of where the Smart Grid concept should be introduced is indoor and outdoor lighting. The lighting in municipal as well as in other buildings is often turned on almost all day neglecting the real need for illumination. The road lighting is also often turned on constantly during the night time without any correction depending on traffic density and weather conditions. If the mentioned parameters were monitored and lighting levels were adjusted then the overall consumption of the lighting system could be reduced.

The main goal of the given work is to identify the hardware required for the development of the energy effective system for the control of lighting. This includes elaboration and analysis of dimmable power converters for luminaries [1], choice sensors and control elements, as well as implementation of communication between various elements of the lighting system (first of the all, between its luminaries and sensors).

\section{Choice and optimization of power supply for LED luminaries}

Power LEDs are low voltage $(\approx 3 \mathrm{~V})$ semiconductor elements. Even connected in series they require a converter (ballast). The light regulation demands an even more sophisticated converter. In case of LEDs, this function requires either current regulator or dimming PWM or group commutation of LEDs.

\section{A. Survey of LED Solutions Available on Market}

At the given time quite a lot of power supply topologies based on integrated circuits (IC) are available for commercial implementation. Some of them are included in Table I. In order to elaborate the optimal power supply it was decided to estimate them from the point of view of energy efficiency and compatibility with Smart Lighting System (SLS).

It has been found that the most of proposed supplies operate as general purpose voltage regulators not intended specifically for LED loads. They are usually built as flyback (1,3 and 5) true buck (4, 7 and 8$)$ or half bridge ( 7 and 9) switch mode or quasi resonant converters. Another configuration is a simple step-down chopper (2 and 6) for a series connection of LEDs and current smoothing coil. From the point of view of the efficiency it seems to be the best (due to the minimal number of components).

Power Factor (PF) of the converters is mostly not controlled or controlled with passive means. Only a few examples provide active PF correction - either with a special IC (8) or with the same chip controlling doublestage (3 and 9) or single-stage unified (5) converter. Of course, these topologies provide a better PF.

From the point of view of compatibility with SLS the most significant feature of the power supply is its dimming method. Mostly it is assumed that the lighting is adjusted by means of the voltage phase cut-off $(1 \ldots 5)$ or with a special PWM signal (6...8). The first approach is mostly intended for existing systems already equipped with triac regulator and is not suitable in SLS. The other method-dimming PWM just enables/disables the converter with rather low frequency (compared to switching frequency). It was experimentally found in [2] such dimming has a lot of drawbacks: lower efficiency, higher temperature of LEDs etc. 
Table I: Some IC based LED ballast

\begin{tabular}{|c|c|c|c|c|c|c|c|c|}
\hline & Manufacturer/IC & Topology & $\begin{array}{l}\text { Power/ } \\
\text { Current }\end{array}$ & $\begin{array}{l}\text { Dimming } \\
\text { method }\end{array}$ & Isolation & $P F C$ & Efficiency & Reference Design \\
\hline 1 & NXP/SSL2101 & Flyback & $\begin{array}{l}\text { Int. } \\
17 W\end{array}$ & Triac & Yes & No & $78 \%$ & UM10341 \\
\hline 2 & NXP/SSL2101 & $\begin{array}{l}\text { Step-down } \\
\text { chopper }\end{array}$ & $\begin{array}{l}\text { Int. } \\
9 W\end{array}$ & Triac & No & No & $66 \%$ & UN10342 \\
\hline 3 & NXP/SSL1750 & $\begin{array}{l}\text { Boost }+ \\
\text { Flyback }\end{array}$ & Ext. & Triac & Yes & $Y e s / 0.95$ & $85 \%$ & UM10321 \\
\hline 4 & $\begin{array}{c}\text { National } \\
\text { Semiconductor/ } \\
\text { LM3445 }\end{array}$ & buck & Ext. & Triac & No & No & - & $R D-172$ \\
\hline 5 & $\begin{array}{c}\text { ON Semiconductor/ } \\
\text { NLC3000 }\end{array}$ & Flyback & Ext. & Triac & Yes & $Y e s / 0.93$ & $82 \%$ & $\begin{array}{c}\text { NCL30000LED2 } \\
\text { GEVB }\end{array}$ \\
\hline 6 & Supertex/HV9910 & $\begin{array}{l}\text { Step-down } \\
\text { chopper }\end{array}$ & Ext. & $P W M$ & No & No & $90 \%$ & $H B 9910 B D B 2(7)$ \\
\hline 7 & $\begin{array}{c}\text { International } \\
\text { Rectifier IRS2541 }\end{array}$ & $\begin{array}{l}\text { Buck, } H- \\
\text { bridge }\end{array}$ & Ext. & $P W M$ & No & $N o$ & $85 \%$ & $A N 1131$ \\
\hline 8 & $\begin{array}{c}\text { STM/ L6562A (PFC } \\
\text { cnt.) }\end{array}$ & $\begin{array}{c}\text { Boost }+ \\
\text { Buck }\end{array}$ & Ext & $P W M$ & No & Yes/0.99 & $90 \%$ & $U 0670$ \\
\hline 9 & $\begin{array}{c}\text { Power Integrations/ } \\
\text { PLC810PG }\end{array}$ & $\begin{array}{c}\text { Boost, } \\
\text { Resonant } \\
\text { H-bridge }\end{array}$ & Ext & - & Yes & Yes/0.97 & $92 \%$ & DER212 \\
\hline
\end{tabular}

\section{B. Practical Configuration of Power Supply for LEDs}

The above mentioned data reveals difficulty to choose a completely integrated solution. For this reason it has been decided to combine an efficient topology of power supply (for example, resonant H-bridge with PLC810) and stepdown chopper as a dimmer (

Fig. 1) that is controlled and protected by a microcontroller (MCU).

The tested luminary is configured of 7 LEDs W724C0 (Seoul Semiconductor, 10W, 2.8A, 3.6V). The overall voltage drop equals $\mathrm{V}_{7 \mathrm{LED}}=7 \times 3.6 \mathrm{~V}=25 \mathrm{~V}$. The margins of the safe operating area roughly define the voltage of the primary supply to $\mathrm{V}_{\mathrm{d}}=40 \mathrm{~V}$ but the parameters of semiconductor switches to $60 \mathrm{~V}$ and $4 \mathrm{~A}$. In order to simplify the cooling system of test-bench better elements have been chosen (IRFZ44N as VT1 and SBL1060 as VD1). At such parameters the chopper has to operate with its duty cycle of $D=25 / 40=0.625$. Then the value of smoothing inductance $\mathrm{L}_{\mathrm{SM}}$ can be calculated based on the value of the maximal ripple of LED current $\Delta \mathrm{I}_{\mathrm{MAX}} \approx 0.3 \mathrm{~A}$ ( $10 \%$ of the rated current), switching period $\mathrm{T}_{\mathrm{S}}=12.5 \mu \mathrm{s}$ :

$\mathrm{L}_{\mathrm{SM}}=\left(\mathrm{V}_{\mathrm{d}}-\mathrm{V}_{7 \mathrm{LED}}\right) \cdot \mathrm{D} \cdot \mathrm{T}_{\mathrm{S}} / \Delta \mathrm{I}_{\mathrm{MAX}}=$

$(40-7 \cdot 3.6) \cdot 0.625 \cdot 12.5 / 0.3=385 \mu \mathrm{H}$

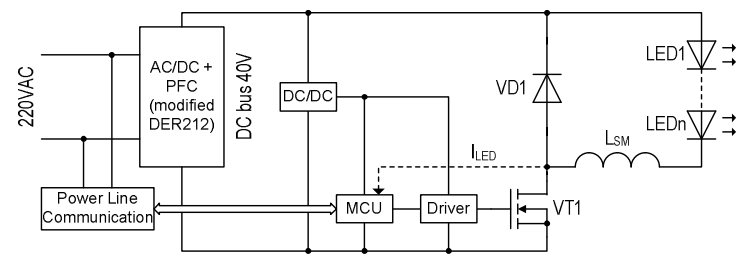

Fig. 1. Functional diagram of the power supply for SLS

\section{Controllability of Chopper}

The described circuit has been assembled and tested. The obtained experimental results, first of the all, show difficulty of regulation. The obtained regulation curve (LED's current versus duty cycle - Fig. 2) basically follows the highly nonlinear Volt-Ampere characteristic of the LED. For this reason in the active regulation region very low changes of the duty cycle lead to very significant changes of the current. At $\mathrm{V}_{\mathrm{d}}=35 \mathrm{~V}$ active regulation span is only $12 \%(55 . .67 \%$ for currents $10 \ldots 90 \%$ of maximal LED's current $3 \mathrm{~A}-0.3 \ldots 2.7 \mathrm{~A})$. A higher supply voltage provides a narrower regulation span. It is obvious that the most functional PWM regulation $(17 \%$ or $78 . .95 \%$ for the same currents $0.3 . .2 .7 \mathrm{~A}$ ) can be achieved if the voltage of the primary supply is close to that of the LEDs $(25 \mathrm{~V})$.

\section{LED @ PWM}

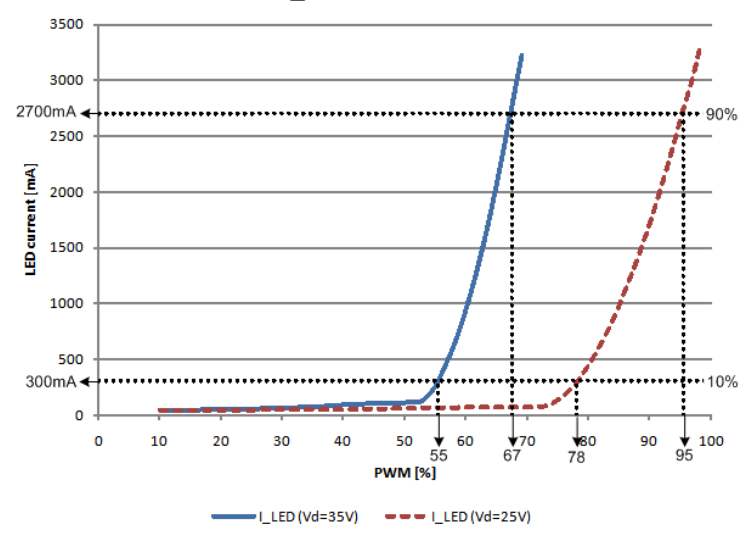

Fig. 2. Measured duty cycles available for dimming 


\section{Rate of Change of Light}

Another significant result of the experiments regards the rate of change of light. It has been found that it is fast enough (Fig. 3 and Fig. 4) to cause stroboscopic and flickering effects. As it can be seen the light intensity (green curve - voltage measured across a photodiode located just under LED) follows the current of LED (red curve) with a delay of few hundred nanoseconds. Therefore a pulse width modulated current produces a distinct pulse width modulated light that leads to the mentioned undesirable effects. The conducted experiments prove that light intensity must be controlled by means of the changes of continuous LED current.

The measurements have been taken for various types of LEDs and different types of ballast (Fig. 3 shows results for the proposed converter while Fig. $4-$ for typical IC based application).

On the other hand, the obtained results confirm that the proposed topology is fast enough and is able to operate in SLS.

\section{Overview of communication chips}

Initial configuration of the proposed application is aimed to study Power Line Communication technology, which allows organizing communication without any new cables. There are various examples of Power Line Communication (PLC) modems for this purpose, which differ by functionality, availability of internal peripherals, implemented modulation method, as well as integrated/supported protocol.

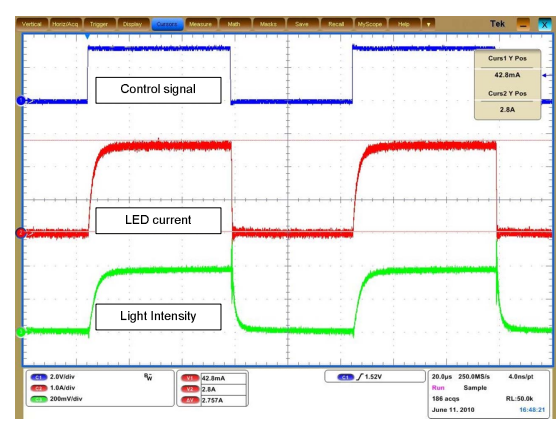

Fig. 3. Light intensity at current PWM in the test-bench

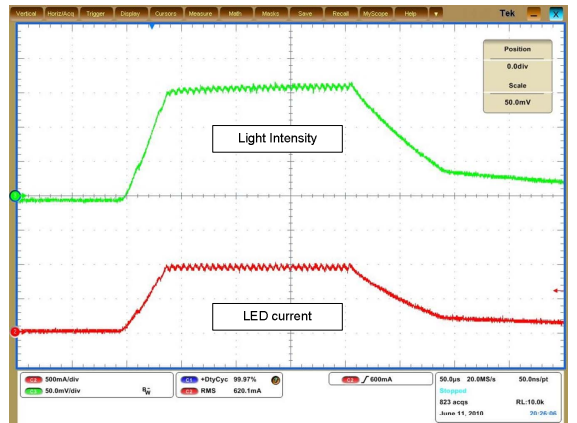

Fig. 4. Light intensity of LEDs driven by IRS2541
Yitran

Yitran company affords several models of Power Line Communication chips: IT700 family allows choosing whether Protocol Controller Architecture version, which requires external host controller with command communication interface over serial bus between them. Next model of IT700 chip is Open Solution Architecture, which allow using all internal peripherals and communication stack building own application, that eliminates the need for an external host controller.

The IT700 is a highly integrated System-on-a-Chip (SoC) powerline communication modem. It incorporates Yitran's reliable Physical Layer (PHY) based on DCSK modulation, high performance Data Link Layer (DLL) and Yitran Network Layer (Y-Net) protocol. The IT700 complies with worldwide regulations (FCC part 15, ARIB and CENELEC bands) of narrowband PLC applications.

Another Yitran family is IT800, which is similar to IT700 model with logical command language over serial interface, except Y-Net layer functionality. This model allows to initialize it in normal operation mode when transferred packet are processed by DLL and PHY layers, or data can be send at once to PHY layer, what means that host controller needs to handles channel access procedure by internal software. IT800D model could be a good choice to develop custom nodes consisting of host processor, which implements or controls the process or the task, and IT800 chip as the power line communication modem.

\section{Echelon}

Echelon company has introduced new automation network structure by integrating communication capability in lowest parts of the automation system and called it Local Operating Network (LON). It consists of sensors and actuators which communicate between each other without a central controller or a PC.

Every node operates with Network Variables (NV). It requests $\mathrm{NV}$ from the network (input $\mathrm{NV}$ ) or can also provide variables for other nodes (output NV). For example, lighting ballast should process input variable of illumination level, to define the brightness of the luminary. At the same time the ballast can provide information about itself: consumed power, current, temperature, working hours - output network variables.

Nodes communicate using LonTalk protocol, developed by Echelon. It offers four basic types of message service: acknowledged; request/response; unacknowledged repeated and unacknowledged, choosing which depends on certain application.

There are several basic power line chips called Smart Transceiver with model number PL3120 and PL3150. They are similar and consist of three processors (media access CPU, network CPU, application CPU), eleven universal inputs/outputs, $2 \mathrm{kB}$ of RAM for buffering network data and variables, $24 \mathrm{kB}$ of ROM which stores firmware (LonTalk protocol) and EEPROM for compiled 
user application code written in Neuron C (4kB for 3120 and external memory IC for PL3150 chip).

These chips requires code to be written in special programming language called Neuron $\mathrm{C}$, which requires specific tool - LBS based device development and debugging software, which is sold in packet with LonMaker (configuration and management of LON) and Lonscanner Protocol analyzer (LonWorks protocol analyzer software). High cost of this software (1900USD) makes it unusable in small projects.

Another way to use Echelon's PLC chips is to connect them to any host controller over SPI or SCI bus and organize communication between their firmware by using Application Programming Interface (API), which helps to use LonTalk functionality at host processor side. Then the application is limited only by host resources.

Fortunately a free software packet is available for building API - ShortStack application development kit, which generates an API code for host controller written in ANSI C, as well as application software for Echelon's chip called ShortStack MicroServer. A special model file should be written before generation process, which contains configuration data of developing node (input and output network variables and etc).

To write the application software to Echelon chip EEPROM memory a versatile flash programmer should be used, which is compatible with PL3120 pinouts or external memory IC pins, when using PL3150 model. Another way to write the application code to the Neuron chip is using special service function of LonTalk protocol, which can rewrite EEPROM remotely. It is useful when the node is already assembled. In this case only network interface is needed (for example USB U20 network interface, which costs about 160USD) and NodeLoader utility, which is included in ShortStack packet. This could be an affordable solution when developing several nodes for test applications.

\section{Selection of the host controller}

The host controller needs to match all requirements that were defined when communication chip and power supply control method were chosen. It would be more preferable if the host controller could also communicate with the illumination sensor, allowing to design one reconfigurable node's board for both - luminary node and sensor node. The final requirements are as follows: SPI or SCI serial interface, at least 200 bytes of RAM and $4 \mathrm{kB}$ of EEPROM (because of API code requirements defined by Echelon), Digital Output (with implemented PWM signal for LED driver control), and analog input or additional serial interface for lighting sensor (for example APDS9300 with $\mathrm{I}^{2} \mathrm{C}$ interface).

TI microcontroller MSP430F2274 has all the necessary peripheral modules for proposed solution it has two adaptive serial interface modules, $1 \mathrm{kB}$ of RAM and $32 \mathrm{kB}$ of Flash memory, which is enough for the proposed solution

\section{Conclusions}

Significant energy consumption of street illumination facilitates the development of new more efficient illumination systems. The solution discussed in the paper is built on the smart LED luminaries with power line communication capability.

Various LED driver integrated circuits are compared by their internal topologies, efficiency, dimming approach, integrated PFC, revealing that the most energy efficient DC converter topology is the step-down chopper due to its minimal count of components and resonant halfbridge due to minimized switching losses.

The simulation results of the chosen LED driver schematics (step-down chopper) with PWM dimming approach have revealed a highly nonlinear LED current dependency on the control PWM duty cycle, that is similar to Volt-Ampere characteristic of LED. This means that it is not the best solution for dimming due to the narrow working band of control parameter.

Implementation of communication over power lines was studied by comparing various PLC chips of different vendors - Yitran and Echelon. Their internal functionality and potential of fast integration were analyzed, as well as the required software and hardware tools were estimated.

The Echelon PL3150 and TI MSP430F2274 host controller with interfaced lighting sensors APDS-9300 have been chosen for further implementation.

Based on the presented analysis a particular configuration of SLS has been developed. The final assembling, testing and analysis of the results are expected soon.

\section{References}

[1] H. van der Broeck; G.Sauerlander and M.Wendt "Power driver topologies and control schemes for LEDs". Applied Power Electronics Conference, (APEC 2007, Anaheim, California), pp. 1319-1325;

[2] I.Galkin, A. Avotinsh, A. Suzdalenko, L. Ribickis. "Comparison and Choice of Supply and Driver Unit for Power LED Luminary" In Proc. RTU $50^{\text {th }}$ Internatinal Conference (Power Electronics and Automation section, RTU, Riga, Latvia, 2009), pp.137-140.

[3] O. Mukhtar, S.M. Golam, E. Liu and T.O. Korhonen "Development of high-rate control system for LED based general lighting applications". Power Line Communications and Its Applications International Symposium (ISPLC 2005, Vancouver, Canada), pp. $417-421$;

[4] LONWORKS Technology Device Data, Rev. 3.1 1997.

[5] IT800D Power Line Communication Modem Data Sheet (IT800-DS-017-R1.6). 